\title{
The Acute Abdomen Decision Making course for the initial management of non traumatic acute abdomen: A proposition of the World Society of Emergency Surgeons
}

\author{
Belinda De Simone, ${ }^{1}$ Luca Ansaloni, ${ }^{2}$ Massimo Sartelli, ${ }^{3}$ Federico Coccolini, ${ }^{2}$ Ciro Paolillo, ${ }^{4}$ \\ Massimo Valentino, ${ }^{5}$ Giorgio Ricci, ${ }^{6}$ Fausto Catena ${ }^{1}$
}

${ }^{1}$ Department of Emergency and Trauma Surgery, University Hospital of Parma, Parma; ${ }^{2}$ Department of Emergency and Trauma Surgery, Bufalini Hospital, Cesena; ${ }^{3}$ Department of General Surgery, Macerata Hospital, Macerata; ${ }^{4}$ Emergency Department, Brescia Ospedale Riuniti, Brescia; ${ }^{5}$ Radiology Unit, Department of Emergency, Parma University Hospital, Parma; ${ }^{6}$ Emergency Department, Verona Hospital, Verona, Italy

\begin{abstract}
The Acute Abdomen Decision Making course ${ }^{\circledR}$ (AADM $\left.{ }^{\circledR}\right)$ was developed by the World Society of Emergency Surgeons (WSES) members with the purpose to teach a systematic and concise method for approaching acute abdomen patient. It was first introduced in 2016. The course explains step by step the clinical reasoning to follow for immediate managing of the acute abdomen in emergency department with a safe and reliable method. We developed this original decision making model AADM following the Advanced Trauma Life Support (ATLS) philosophy in evaluating non-traumatic abdominal pain patient. The A-B-C-D-E-F management of non-traumatic acute abdominal pain is conceived by WSES to make easy and systematic the evaluation of patients in Emergency Department. AADM course teaches how to assess an acute abdomen patient's diagnosis and determine the therapeutic approach to reduce unnecessary exams and hospitalizations.
\end{abstract}

Correspondence: Belinda De Simone, Department of Emergency and Trauma Surgery, University Hospital of Parma, via Gramsci 15, 43100 Parma, Italy.

Tel.: +39.320.0771984.

E-mail: desimone.belinda@gmail.com

Key words: Acute abdomen; Educational program; Clinical practice; Abdominal pain; Emergency department.

Contributions: FC and BDS conceived and design the study; BDS collected the data; FC and BDS wrote the manuscipt; LA, MS, FCo, CP, $\mathrm{MV}$, GR read, revised and approved the manuscript.

Conflict of interest: the authors declare no potential conflict of interest.

Funding: none.

Received for publication: 14 August 2018.

Revision received: 10 November 2018.

Accepted for publication: 11 December 2018.

This work is licensed under a Creative Commons Attribution 4.0 License (by-nc 4.0).

(C) Copyright B. De Simone et al., 2019

Licensee PAGEPress, Italy

Emergency Care Journal 2019; 15:7767

doi:10.4081/ecj.2019.7767

\section{Introduction}

The term acute abdomen (AA) defines a clinical syndrome characterized by the sudden onset of severe abdominal pain requiring emergency medical or surgical treatment. It is the most common cause of admission in emergency department (ED).

Various potentially life-threatening diseases can cause AA, thus a rapid and accurate diagnosis is essential to reduce morbidity and mortality.

In an analysis of more than 10,000 patients presenting with non traumatic acute abdominal pain (AAP), aetiology could not be determined in one-third of these cases; of those patients in whom a diagnosis was made, $28 \%$ had appendicitis, $9.7 \%$ acute cholecystitis, $4.1 \%$ small bowel obstruction, $4 \%$ acute gynecological disease, $2.9 \%$ acute pancreatitis, $2.9 \%$ acute renal colic, $2.5 \%$ perforated peptic ulcer, and $1.5 \%$ acute diverticulitis. ${ }^{1}$

In ED, health professionals often manage medical problems in critical situations, under time pressure and on the basis of vague information. Moreover clinical features of patients with AA can be unspecific and challenging, consequently it can be very difficult to differentiate acute life-threatening disease from non-specific abdominal pain (NSAP) with a high level of diagnostic accuracy in a minimal period of time by a careful and quick patients evaluation, avoiding unnecessary further diagnostic activities.

Physical examination and laboratory exams are often non-specific, and clinical presentation of many entities overlaps. Often diagnostic and efficient imaging evaluations are indispensable.

Computed tomography (CT) has gained widespread acceptance as the first-line imaging modality in patients presenting with acute abdominal pain..$^{2-5}$ It is the most time-effective and accurate imaging technique, and, if the working clinical diagnosis is incorrect, CT provides sufficient information for an alternative diagnosis. The introduction of multi-detector CT has yielded additional advantages, which enhance the role of CT in patients with acute abdominal pain. ${ }^{6,7}$ However it is not possible to perform a CT scan in all patients with AA (problem of irradiation in female pregnant patients and in children; administration of contrast medium, contro-indicated in case of acute or chronic kidney failure and allergy). AA can be a challenge in ED and physicians have to manage it. The World Society of Emergency Surgeons (WSES) Educational Team decided to develop a simply decision making method to help physicians in this clinical, high complex, reasoning, considering the dual process theory that has provided a framework of cognitive processes to assist doctors in developing clinical reasoning skills, critical especially under high workload and ele- 
vated stress levels. ${ }^{8,9}$ In this paper we present the steps (A-B-C-DE-F) of the Acute Abdomen Decision Making model (AADM) for the management of non traumatic acute abdominal pain proposed by WSES (Table 1).

\section{Methods of research}

We performed a literature research for relevant scientific articles on the management of AA, clinical reasoning and academic educational programs. We used the following search terms: acute abdomen, non-specific abdominal pain, acute abdominal pain, abdominal pain in emergency department, teaching quality improvement, academic educational program, clinical reasoning We considered and evaluate all scientific papers published between 2007-2017. We developed an effective decision making method to manage the patient presenting with non traumatic acute abdominal pain and we report it.

\section{The "A-B-C-D-E-F" management of non-traumatic abdominal pain}

\section{Step 1: A as Anamnesis}

You may to investigate the causes of AA asking the right questions, focusing patient's answer on:

i) previous abdominal and non-abdominal diseases;

ii) previous surgical interventions: look at the abdomen and check for the presence of scars;

iii) symptom pain: onset, location/radiation, progression, character, intensity;

iv) accessory symptoms as fever, vomiting, bowel movements, diarrhea or constipation;

v) medications;

vi) feeding;

vii) presence of allergy;

viii) presence of urination;

ix) pain control by medications or not

In all female child bear patients admitted for AA, you may obtain a menstrual history. You can collect patient's effective medical history using the multiple choice form and pain control showed in Table 2.

\section{Step 2: B as Body examination}

You have to focus body examination on palpation, putting aside the classical clinical body examination described in all medical textbooks. You may note pain location, its degree and the presence or not of peritonism's signs, on a map-score system showed

Table 1. The Acute Abdomen Decision Making course ${ }^{\circledR}$ steps.

\begin{tabular}{|c|c|}
\hline A - Anamnesis & $\begin{array}{l}\text { Collect an effective medical history using } \\
\text { multiple choice forms and pain control }\end{array}$ \\
\hline B - Body Examination & $\begin{array}{l}\text { Doing a standardized patient exam, filling the } \\
\text { abdominal pain and peritonism map and score }\end{array}$ \\
\hline C - Clinical Ultrasound & $\begin{array}{l}\text { Doing an office ultrasound to evaluate aorta, } \\
\text { gallbladder, bladder, kidney }\end{array}$ \\
\hline D - Do or not & $\begin{array}{l}\text { Do or not Laboratory Tests and insert } \\
\text { or not foley catheter and/or nasogastric tube }\end{array}$ \\
\hline $\begin{array}{l}\text { E - Evaluate your } \\
\text { working diagnosis }\end{array}$ & $\begin{array}{l}\text { Evaluate your working diagnosis } \\
\text { (consider age and sex) }\end{array}$ \\
\hline $\begin{array}{l}\text { F - Follow guidelines } \\
\text { or follow up }\end{array}$ & $\begin{array}{l}\text { Follow guidelines according your working } \\
\text { diagnosis, and follow-up the case if } \\
\text { diagnosis is unclear }\end{array}$ \\
\hline
\end{tabular}

in Table 3. Making a systematic abdominal examination, split the abdomen in 4 major quadrants: the upper right and left quadrants and the lower right and left quadrants. This will allow you to more easily study abdominal regions. As you can see in Figure 1, in this way you can imagine the organs contained in each quadrant and generate your diagnostic hypothesis.

Localized tenderness is usually a reliable evidence of underlying disease (localized peritonitis): right upper quadrant tenderness may be caused by cholecystitis, ulcer disease, pancreatitis, or hepatitis; epigastric tenderness is usually due to pancreatitis or peptic ulcer disease; right lower quadrant tenderness may be related to appendicitis, cecal diverticulitis, or perforated carcinoma, crohn disease whereas left lower quadrant tenderness is usually due to sigmoid diverticulitis or perforated carcinoma. Flank tenderness is usually related to renal disease. When tenderness is generalized, consider causes for generalized peritonitis. Rectal examinations as vaginal examination are not recommended in routine abdomen examination because they are sometimes distressing for the patient. A rectal examination may be indicated when it is necessary (to check presence of stools - fecaloma in bowel obstruction; melena or rectorrhagia). The vaginal examination has to be performed by a gynecologist when extrauterine pregnancy or gynecologic pathology, including pelvic inflammatory disease (PID), is suspected: pain upon movement of the cervical canal and appendicular tenderness on internal examination may be useful in the diagnosis.

Table 2. A as Anamnesis: collect the medical history using the multiple choice form and pain control.

\begin{tabular}{ll} 
1. & previous diseases \\
2. & previous surgical interventions \\
- brain \\
- neck \\
- thorax \\
- limbs \\
- abdomen (scars?) \\
\hline 3. & allergy \\
4. & urine \\
\hline 5. & feeding \\
6. & vomit \\
\hline 7. & stool-gas \\
8. & pain control \\
\hline 9. & drugs
\end{tabular}

Table 3. B as Body examination: acute abdominal pain/peritonism map (APPM) score.

$\begin{array}{ccc}\text { Abdominal quadrants } & \text { Pain } & \text { Peritonism APPM score } \\ & (x) \text { low } & (x) \text { low } \\ & (x \times) \text { mild } & (x \times) \text { mild } \\ & (x \times x) \text { high } & (x \times x) \text { high }\end{array}$

Upper right quadrant (RUQ)

Lower right quadrant (RLQ)

Upper left quadrant (LUQ)

Lower left quadrant (LLQ)

APPM score: $<2$ non-specific abdominal pain; $>2<5$ suspected surgical disease; $>5$ high probability emergency surgical disease. 


\section{Step 3: C as Clinical ultrasound}

Ultrasound (US) abdominal examination is widely available and easy accessible as first line imaging investigation to evaluate patients admitted in ED with AA. Well-trained residents and physicians can use tailored US examination to confirm diagnostic hypothesis at the patient's bedside, without waiting for radiologists' availability. By US examination, emergency doctor has the possibility to correlate the US findings with the point of maximal tenderness. He/she can check signs of cholecystitis associated with gallstones; appendicitis (non compressible appendix with a rim of peri-appendiceal fluid) or adeno-mesenteritis (multiple enlarged lymph nodes some of them disposed as a chain in the mesentery), in differential diagnosis with appendicitis; if bladder is full and urinary obstruction caused by renal or ureteral stones. ${ }^{10}$

Point-of-care ultrasonography (POCUS) is defined as a goaldirected, bedside ultrasound examination performed by a healthcare provider to answer a specific diagnostic question or to guide performance of an invasive procedure. ${ }^{11}$ It has the advantages to clarify uncertain findings of the physical exam at the bedside of the patient, identify acute abdominal diseases, or provide image guidance that improves the success and safety of many procedures in the acute care setting.

The examiner's goal is to rule in or rule out a specific condition or answer a "yes/no" question. We have to distinguish the POCUS examination from the imaging consultant's examination. The consultative sonographic exam aims to systematically map out normal and disordered anatomy, assess function and dysfunction in the body or provide guidance for a wide range of interventional procedures. $^{11}$

The most common US technique used to examine patients with AAP is the graded-compression procedure: with this technique, interposing fat and bowel can be displaced or compressed by means of gradual compression to show underlying structures. Furthermore, if the bowel cannot be compressed, the non-compressibility itself is an indication of pathology (inflammation such as appendicitis, intussusception, malignancy or luminal distension resulting from obstruction). ${ }^{12,13}$

Curved (3.5-5.0-MHz) and linear (5.0-12.0-MHz) transducers are most commonly used, with frequencies depending on the application and the patient's stature, on the depth of the anatomical structures and on the aim of the study. Color and power Doppler imaging supplement the information provided by gray-scale imaging, with increased vascularity showed in a number of inflammatory, infectious or neoplastic diseases. ${ }^{13,14}$ Several studies have evaluated the diagnostic accuracy of abdominal US in AA and have found high sensitivity and specificity. ${ }^{15-17}$

Nural et al. ${ }^{15}$ analyzed US findings among 300 patients who presented with AAP; US revealed a different diagnosis than the clinical impression in $69(23 \%$; 95\%CI, 18.2- 27.7\%), and confirmed the diagnosis in 121 (40\%; 95\% CI, 34.4-45.5\%) patients. The US changed the treatment plans in $47 \%$ (95\%CI, 41.3-52.6\%) of the patients. When US results were compared with the discharge diagnosis, there was concordance in $238(79.3 \%$; 95\%CI, 74.3$83.6 \%)$ patients but not in $62(20.6 \% ; 95 \%$ CI, $16-25.1 \%)$. Among 121 patients the initial clinical impression agreed with the US diagnosis and there was concordance with the discharge diagnosis in $105(86.7 \%$; 95\%CI, 80-92.7\%). Nural et al. reported that statistically the concordance of US findings with the discharge diagnosis was significantly higher than that of the initial clinical impression. ${ }^{15}$ The great disadvantage of US is the possibility of interexaminer variability. A survey on the use of US in non-trauma patients was conducted, targeting all emergency physicians and emergency medicine trainees in a single adult tertiary referral cen- tre with the aim to highlight how many non-radiologist physicians are able to do a diagnostic US abdominal examination. The survey showed that a course in non-trauma US had been completed by $58 \%$ of respondents. The most common non-trauma formal US training was in vascular access $(82 \%, 95 \%$ confidence interval $[\mathrm{CI}]$ 66.8-90.6), detection of abdominal aortic aneurysm $(79 \%, 95 \% \mathrm{CI}$ 63.7-88.9) and pericardial fluid (84\%, 95\% CI 69.6-92.6). Most doctors felt the greatest barriers to the use of US in the non-trauma patient were the lack of teaching, confidence in findings, experienced supervisors and time. The authors concluded that among Emergency Department personnel, use of US to diagnose several non-traumatic conditions was low. Specific training was always associated with significantly more US use. Increased training and availability of US-experienced supervisors might further improve utility of this important adjunct to the practice of emergency medicine. $^{16}$

\section{Step 4: D as Do or not laboratory test and insert or not Foley catheter and nasogastric tube}

You may think to insert a nasogastric tube, if you suspect an intestinal obstruction to avoid vomiting and decrease inhalation's risk; to aspirate gastric fluids and reduce abdominal pain and distention, before asking for imaging and laboratory exams. If you found a full bladder at the US examination, you may insert a Foley's catheter: urinary retention can be cause of AAP and/or paralytic ileus, above all in elderly people.

Laboratory tests that give an indication of systemic illness are clinically important for patients with AAP, when an underlying surgical disease is suspected. ${ }^{17}$ The hCG serum should be always measured in young female patients, admitted for AAP, needing for further explorations. There are significant limitations of imaging and laboratory studies in the evaluation of AAP in fact all diagnostic tests have a false-negative rate. If the history and physical examination leads to a high pre-test probability of a disease, a negative test cannot exclude the diagnosis. ${ }^{17}$ The usefulness of a given test is often evaluated by its ability to rule in or out a given disease process.

In ED our aim is to distinguish AAP caused by potentially sur-

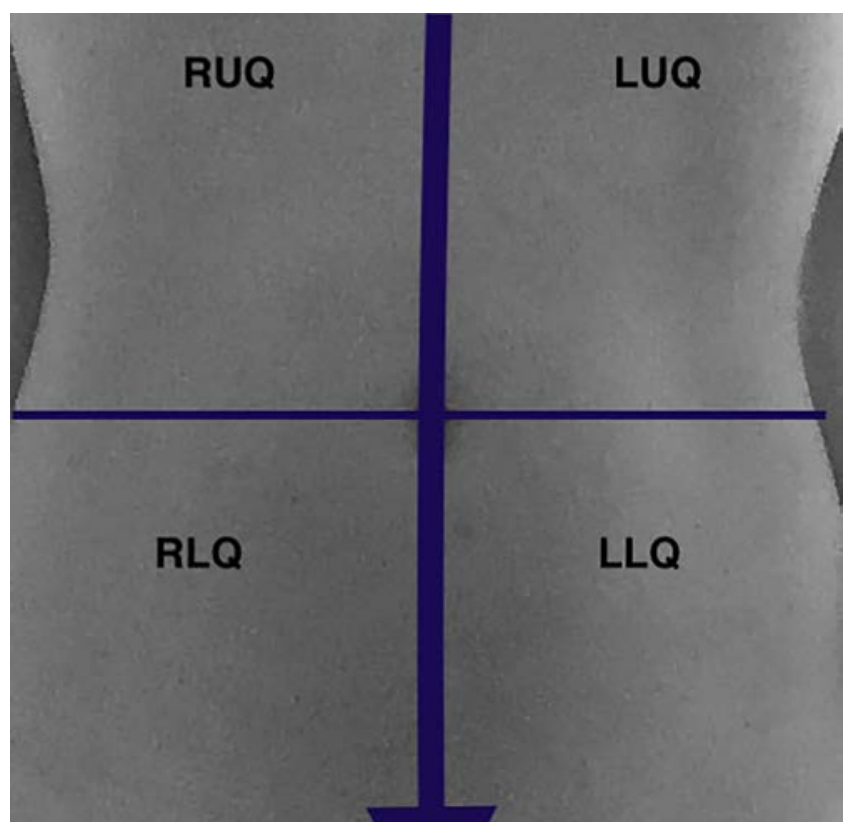

Figure 1. Abdominal quadrants. 
gical diseases as appendicitis, generalized peritonitis, cholecystitis, and bowel obstruction, from NSAP. Panebianco et al. ${ }^{17}$ found that the most common laboratory tests ordered in the ED by percentage of patient visits are complete blood count $(34.0 \%)$, blood urea nitrogen/creatinine (20.1\%), electrolytes (19.1\%), cardiac enzymes $(19.0 \%)$, and liver function tests $(11.5 \%)$. Urinalysis was ordered in $20.2 \%$ of ED visits. In differentiating urgent diseases from specific diseases underlying AAP, Goransson reported that the diagnostic accuracy values of C-reactive protein (CRP) and white blood cell count (WBC) can be elevated. ${ }^{18}$ For $\mathrm{CH}$, CRP has a moderate sensitivity (79\%) and low specificity (64\%) for an urgent diagnosis in patients with abdominal pain at the ED. ${ }^{19}$ Lipase and amylase are elevated in $13 \%$ of patients with other than pancreatic conditions. In $1-2 \%$ of patients, levels of lipase and amylase are elevated more than thrice their reference values. ${ }^{20}$

CRP and WBC count alone are insufficient to differentiate urgent from non-urgent conditions. When clinically non-urgent condition is suspected but the CRP is above $100 \mathrm{mg} / \mathrm{l}$ or the WBC count is above $15 \times 10^{-9} / 1$, the suspicion of an urgent condition rises and additional imaging is warranted. Initially, on presentation at the ED, only CRP and WBC count should be determined. Other laboratory tests can be determined based on the suspicion of a specific diagnosis after medical history and physical examination. ${ }^{21}$

\section{Step 5: E as Evaluate your working diagnosis (consider age and sex)}

At this point, if you followed all the steps till here, you have all clinical, laboratory and imaging information to elaborate your diagnosis of:

i) Potentially emergency surgical diseases:

- Acute cholecystitis

- Acute appendicitis

- Bowel ischaemia

- Abdominal Aortic Aneurysm

- Adhesive small bowel obstruction

- Large bowel obstruction

- Acute diverticulitis

- Perforated peptic ulcer

ii) Not surgical abdominal diseases:

- Renal colic

- Acute pancreatitis

- Acute hepatitis

- Pelvic inflammatory disease

- Gastroenteritis

- Fecal impaction

- Non specific abdominal pain

The incidence of each of these diseases varies according to age and sex. Re-evaluate your working diseases considering age and gender of your patient because epidemiological distribution of surgical abdominal diseases may change.

In a recent retrospective Italian study investigating the epidemiology of AAP in an adult population admitted to an urban ED, the most frequent cause of admission was NSAP. This study reported that biliary colic/cholecystitis, and diverticulitis are more prevalent in patients aged $>65$ years. Appendicitis and renal colic are more frequent in patients aged $<65$ years. NSAP is the most common cause in both age classes, despite being slightly more frequent in younger patients. Renal colic is the most frequent cause of ED admission in men, whereas NSAP was more prevalent in women. Urinary tract infection was higher in women. ${ }^{22}$

Fagerström and his group performed a retrospective cross-sectional cohort study to evaluate the diseases causing AAP during a 26-year period in a ED. They reported that the most common causes of AAP are NSAP, acute appendicitis, biliary disease, bowel obstruction, acute pancreatitis and acute diverticulitis, in this order. ${ }^{23}$

Furthermore the female gender, elderly and immunocompromised patients represent a special population from a diagnostic point of view in the clinical suspicion of acute abdomen. AAP in women may be related to pathology in the pelvic organs. Ectopic pregnancy, pelvic inflammatory disease, and hemorrhagic ovarian cysts are the most commonly diagnosed gynaecologic conditions presenting with acute pelvic pain. Other causes to consider include endometriosis, and postpartum causes such as endometritis, or ovarian vein thrombosis. Finally, non-gynaecologic conditions may overlap in their presentation of acute pelvic pain and should also be considered; the most important of these is acute appendicitis. Often differential diagnosis is made intraoperatively..$^{10-21}$

Older patients represent a particular diagnostic challenge because of clinical features of AAP and laboratory testing often do not reflect the patient condition causing delayed or missing diagnosis with consequently increasing in mortality and morbidity rates. Furthermore, sometimes elderly patient can't express himself because of frailty and dementia. In your diagnostic hypotheses, in these cases you have to consider the abdominal aortic aneurysm, occult urinary tract infection, perforated viscus, diverticulitis and ischemic bowel disease that could be potentially fatal conditions. ${ }^{24}$ Urgent hospitalization and surgical intervention are often required for elderly people complaining of abdominal pain. ${ }^{25}$

Mortality rates increase with patient age..$^{24,25}$

Gardner and his colleagues retrospectively analyzed data about 464 patients aged $\geq 80$ years (mean 89 years, range $80-100$ ), who presented to the ED with acute abdominal symptoms and underwent $\mathrm{CT}$ to assess clinical impact of $\mathrm{CT}$ in elderly patients. ${ }^{26} \mathrm{CT}$ was positive in $55 \%$ of cases. The most common diagnoses were small bowel obstruction, followed by diverticulitis, non-ischemic vascular-related emergency, bowel ischemia, appendicitis, and colonic obstruction. These diagnoses were clinically unsuspected prior to $\mathrm{CT}$ in $43 \%(\mathrm{P}<0.05)$, with significant difficultly in diagnosing small bowel obstruction $(\mathrm{P}<0.05)$, diverticulitis $(\mathrm{P}<0.01)$, and colonic obstruction $(\mathrm{P}<0.01)$. Positive $\mathrm{CT}$ results influenced treatment plans in $65 \%$, surgical in $48 \%$, and medical in $52 \%{ }^{26}$

If the clinical symptoms and signs are weak, laboratory tests and imaging should be used in synergy to evaluate the patients carefully. If the calculated risk with the map score, considering imaging and laboratory results is high for a potentially surgical abdominal disease, ask for a CT; if it is low, you can wait and reevaluate the clinical evolution of the patient. In case of obese and elderly patients, physical examination becomes difficult also for experienced physicians, and imaging studies as CT of the abdomen and the pelvis, should be requested to do not delay diagnosis. Several authors are agree to affirm that utilization of abdominopelvic CT in geriatric patients presenting to the ED with acute abdominal symptoms strongly improve ED diagnosis accuracy and appropriate management of the patient, decreasing delay in surgical treatment. ${ }^{27-30}$ Don't forget this: you may not searching for diagnosis requiring a series of laboratory exams and imaging but to develop a working diagnosis to confirm your diagnostic hypothesis requiring the right laboratory and radiological exams. It is fundamental to develop a working diagnosis to decrease unnecessary and misleading exams.

\section{Step 6: F as Follow international guidelines according to your working diagnosis and follow up if diagnosis is unclear}

According to your strong diagnostic hypothesis, check international available guidelines as for example WSES guidelines, for 
diagnosis and management of disease causing AAP that you hardly suspect and follow recommendations. ${ }^{31}$ If diagnosis and clinical features are not clear and the patient is stable, you can treat the pain and confirm hospitalization for clinical surveillance in an observation unit or in the ED and check eventually for disease progression. Keep the patient fasting and don't start antibiotics if you don't know what you are treating. If the patient is young without co-morbidities and AAP is controlled after the administration of first-line painkillers as paracetamols, you can discharge home the patient and give him the instructions to return if the pain worsens, new vomiting or fever occurs, or if the pain persists beyond 8-12 hours. Such instructions are targeted at ensuring the return of a patient who has progressed from an early appendicitis or small bowel obstruction, the two most common surgical entities erroneously discharged from an ED.

The NSAP usually disappears within 2-3 days, and in the majority of cases disappears after 2-3 weeks. However, a disease requiring surgery might be diagnosed subsequently, and a careful follow-up is necessary in all doubtful cases. ${ }^{32}$

The axiom of the patient, not the test certainly applies in the patient with AAP. An unexpected negative test result should prompt a reassessment of the patient and consideration for observation to repeat examination for disease progression.

\section{Discussion}

AADM is a simple and reproducible method to manage AAP in ED and decrease a large number of negative work-up, unnecessary surgeries, health costs. AA represents the cardinal symptom behind a vast number of possible underlying diseases, the challenge remains to early identify patients with an indication for emergency surgery. Clinical reasoning skills are critical in emergency situations because of stress under time pressure. Clinical algorithms apply to complex disease as AAP help physicians to systematically consider clinical, to clearly work out diagnostic hypothesis and follow them to confirm the origin of non-traumatic AAP. In developing an efficacy AADM method, it is important to understand the underlying cognitive processes at the basis of the problem solving process. Clinical reasoning or medical problem solving is known to involve analytical thought processes and continuously improved intuition, based on clinical experience. The dual-process theory proposes a hypothetical model to understand how these systems interact. ${ }^{8,9}$ Pattern recognition in the shortest time is the starting point for processing, it leads to the diagnosis intuitively and quickly by unconscious, memory-based, and parallelized processing. ${ }^{8}{ }^{8}$ Unrecognized patterns must be consciously analyzed piece-by-piece (analytical thought), until finally intuitive processing is possible or the purely rational diagnosis is made..$^{8,9}$ The script theory focuses on knowledge organization as the foundation of clinical reasoning during patient encounters. It proposes an explanation for how information is stored in and retrieved from the human mind to influence individuals' interpretation of events in the world. Each time it processes a scene, the brain begins by retrieving relevant prior knowledge from memory and using it as a basis for building a model, or representation, of the perceived object or event. It then relies on this model to make predictions about what information it should be receiving from the world. Since interpretation depends heavily on prior knowledge, it stands to reason that the composition and structure of an individual's scripts are pivotal for influencing which signals he attends to and how he acts within the world. ${ }^{8}$ Studies of expertise development in medicine have consistently shown that those considered to be experts are distinguished not by their superior problem-solving skills but by the content and organization of their knowledge base. According to script theory, medical knowledge is bundled into networks called illness scripts that allow physicians to integrate new incoming information with existing knowledge, recognize patterns and irregularities in symptom complexes, identify similarities and differences between disease states, and make predictions about how diseases are likely to unfold. These knowledge networks become updated and refined through experience and learning..$^{8,9}$

Illness scripts elaboration offers an explanation of how experts and novices differ from each other. In clinical practice experts use significantly less of their biomedical knowledge than young physicians to explain medical procedures. ${ }^{8}$

The AADM course was conceived by WSES Educational task force with the aim to guide clinical reasoning of ED physicians throughout the different steps of the early approach to patients presenting with AAP.

The main objective is decreasing delay in diagnosis and treatment of the abdominal diseases underlying acute abdomen by improving know-how and the decision making skills necessary for daily practice. The course consists in one-day full session designed in a interactive format.

The course is divided in 2 parts: a theoretical session and case scenarios interactive-discussion. We proposed a methodology simple and reproducible such as the Advanced Trauma Life Support (ATLS).

ATLS was developed in the United States in the 1970s to provide a standardized method for the initial assessment and treatment of severely injured victims by physicians working in ED. The concept, based on the principles treat first what kills first and do no further harm, was initially meant for doctors who are not already experienced with care of major trauma victims.

The aim of ATLS course is to improve the initial survival of trauma patients through treatment protocols and by a systematic method. According to ATLS philosophy, AADM course was conceived to make systematic and simple the evaluation of AAP in ED by ED physicians. Each step of the clinical reasoning is indicated by one letter of the alphabet as for the basic life support educational method. An early working diagnosis results in more accurate management of patients presenting non traumatic AA and it leads to better outcomes.

Patients with acute general surgical conditions often present with complex problems, may deteriorate quickly and are prone to complications. The spectrum of these illnesses which range from acute appendicitis and cholecystitis to intestinal obstruction, mesenteric ischemia and other forms of abdominal sepsis, requires prompt and comprehensive efforts of well trained surgeons, engaged multidisciplinary teams and streamlined health care systems. The development of surgical education is a universal priority to obtain the best management of patients with AA and improve patients outcomes. The golden hour concept of trauma care has lead to the development of trauma teams. The education of acute care surgeon has been always a complex processus because acute care surgery includes surgical critical care and the surgical management of acutely ill patients with a variety of conditions including trauma, burns, surgical critical care or an acute general surgical condition. Two organizations, the American College of Surgeons and the American Association for the Surgery of Trauma have been the first to invest in the expertise of the acute care surgeon improving education of general surgeons. The concept spreaded was treatment should begin without delay and advances in medicine are often a function of advances in trauma... and when trauma care has 
made quality advances, medicine and society have benefited. ${ }^{33}$

In Europe there is a great variation in the way trauma and nontrauma emergency surgery is organized. Currently 3 trends in trauma care can be observed in Europe. The first one follows the past United States model with trauma systems and trauma surgery based education (ATLS, regionalization of trauma care, trauma centers). The second one aims to integrate trauma care with nontrauma emergency surgery, such as the acute care surgery model in the United States. The third option is based on the past orthopedic surgeon dominated trauma surgery model with visceral and vascular injuries managed either by broadly trained trauma orthopedic surgeons, or visceral specialists under the coordination and leadership of orthopedic surgeons. ${ }^{34-36}$

AADM course was conceived with the aim to prepare residents and physicians for the current emergency department as it now exists: stressful working conditions, overcrowding, delay in the management of patients. The patient admitted in ED will not move immediately to ward beds and he needs rapid initial assessment and treatment in the shortest possible time. Reviewing the recent literature, in Europe there are many courses in emergency surgery, that refers to acute general surgical disorders, focused above all on in the development of surgical skills. The AADM is conceived to improving the initial management of non-traumatic AA patients in $\mathrm{ED}$, by an original educational format.

\section{Conclusions}

Academic medical education program need to clear action to reach a solid preparation of residents, often alone at night, and ED physicians. The aim of WSES is to revolutionize traditional academic education of physicians. Theory is important in medicine but it can be different from what we can find approaching patients every days. WSES proposes the AADM course as an effective and simple decision making model to approach and systematically manage patients presenting AAP in ED in the shortest possible time, limiting negative and expensive work-out. It was developed by experienced surgeons and emergency physicians based on daily clinical practice and reviewing available literature, considering all the aspects of clinical reasoning.

\section{References}

1. Shah S. An update on common gastrointestinal emergencies. Emerg Med Clin North Am 2013;31:775-93.

2. Radwan RW, Tang AM, Beasley WD. Computed tomography as a first-line investigation for elderly patients admitted to a surgical assessment unit. Ann R Coll Surg Engl 2018;100:2859.

3. Stoker J, van Randen A, Laméris W, Boermeester MA. Imaging patients with acute abdominal pain. Radiology 2009;253:31-46.

4. Rosen MP, Sands DZ, Longmaid HE 3rd, et al. Impact of abdominal CT on the management of patients presenting to the emergency department with acute abdominal pain. AJR Am J Roentgenol 2000;174:1391-6.

5. Modahl L, Digumarthy SR, Rhea JT, et al. Emergency department abdominal computed tomography for nontraumatic abdominal pain: optimizing utilization. J Am Coll Radiol 2006;3:860-6.

6. Leschka S, Alkadhi H, Wildermuth S, Marincek B. Multi- detector computed tomography of acute abdomen. Eur Radiol 2005; 15:2435-47.

7. Marincek B. Nontraumatic abdominal emergencies: acute abdominal pain: diagnostic strategies. Eur Radiol 2002;12:2136-50.

8. Lubarsky S, Dory V, Audétat MC, et al. Using script theory to cultivate illness script formationand clinical reasoning in health professions education. Can Med Educ J 2015;6:e61-70.

9. Dolmans DH, De Grave W, Wolfhagen IH, van der Vleuten CP. Problem-based learning: future challenges for educational practice and research. Med Educ 2005;39:732-41.

10. Rosen MP, Ding A, Blake MA, et al. ACR Appropriateness Criteria ${ }^{\circledR}$ right lower quadrant pain—suspected appendicitis. J Am Coll Radiol 2011;5:749-55.

11. Bhagra A, Tierney DM, Sekiguchi H, Soni NJ. Point-of-care ultrasonography for primary care physicians and general internists. Mayo Clin Proc 2016;91:1811-27.

12. Dubuisson V, Voïglio EJ, Grenier N, et al. Imaging of non-traumatic abdominal emergencies in adults. J Visc Surg 2015;152:S57-64

13. Mazzei MA, Guerrini S, Cioffi Squitieri N, et al. The role of US examination in the management of acute abdomen. Crit Ultrasound J 2013;5:S6.

14. Dhillon S, Halligan S, Goh V, et al. The therapeutic impact of abdominal ultrasound in patients with acute abdominal symptoms. Clin Radiol 2002;57:268-71.

15. Nural MS, Ceyhan M, Baydin A, et al. The role of ultrasonography in the diagnosis and management of non-traumatic acute abdominal pain. Intern Emerg Med 2008;3:349-54.

16. Lokuge A, Mitra B, Bystrzycki A. Use of ultrasound for nontrauma patients in the emergency department.Emerg Med Australas 2013;25:213-8.

17. Panebianco NL, Jahnes K, Mills AM. Imaging and laboratory testing in acute abdominal pain. Emerg Med Clin North Am 2011;29:175-93.

18. Goransson J, Larsson A. Plasma levels of C reactive protein in the diagnosis of acute abdominal disease. Surge Res Comm 1991;11:107-18.

19. Chi $\mathrm{CH}$, Shiesh SC, Chen KW, et al. C-reactive protein for the evaluation of acute abdominal pain. Am J Emerg Med 1996;14:254-6.

20. Chase CW, Barker DE, Russell WL, Burns RP. Serum amylase and lipase in the evaluation of acute abdominal pain. Am Surg 1996;62:1028-33

21. Gans SL, Pols MA, Stoker J, Boermeester MA, Expert Steering Group. Guideline for the diagnostic pathway in patients with acute abdominal pain. Dig Surg 2015;32:23-31.

22. Cervellin G, Mora R, Ticinesi A, et al. Epidemiology and outcomes of acute abdominal pain in a large urban Emergency Department: retrospective analysis of 5,340 cases. Ann Transl Med 2016;4:362.

23. Fagerström A, Paajanen P, Saarelainen H, Ahonen-Siirtola M, et al. Non-specific abdominal pain remains as the most common reason for acute abdomen: 26-year retrospective audit in one emergency unit. Scand J Gastroenterol 2017;28:1-6.

24. Bugliosi TF, Meloy TD, Vukov LF. Acute abdominal pain in the elderly. Ann Emerg Med 1990;19:1383-6.

25. Lyon C, Clark DC. Diagnosis of acute abdominal pain in older patients. Am Fam Physician 2006;74:1537-44.

26. Gardner CS, Jaffe TA, Nelson RC. Impact of CT in elderly patients presenting to the emergency department with acute abdominal pain. Abdom Imaging 2015;40:2877-82.

27. Graff LG 4th, Robinson D. Abdominal pain and emergency 
department evaluation. Emerg Med Clin North Am 2001;19:123-36.

28. Martinez JP, Mattu A. Abdominal pain in the elderly. Emerg Med Clin North Am 2006;24:371-88.

29. Millet I, Sebbane M, Molinari N, et al. Systematic unenhanced CT for acute abdominal symptoms in the elderly patients improves both emergency department diagnosis and prompt clinical management. Eur Radiol 2017;27:868-77.

30. Lehtimäki TT, Valtonen H, Miettinen P, et al. A randomised clinical trial of routine versus selective CT imaging in acute abdomen: Impact of patient age on treatment costs and hospital resource use. Eur J Radiol 2017;87:1-7.

31. World Society of Emergency Surgeons (WSES). Guidelines. Available from: https://www.wses.org.uk/guidelines
32. Morino M, Pellegrino L, Castagna E, et al. Acute nonspecific abdominal pain: A randomized, controlled trial comparing early laparoscopy versus clinical observation. Ann Surg 2006;244:881-8.

33. Britt LD. Trauma: still the cornerstone of acute care surgery specialty. J Am Coll Surg 2018;226:211-22.

34. Søreide K. Trauma and the acute care surgery model—should it embrace or replace general surgery? Scand J Trauma Resusc Emerg Med 2009; 17:4.

35. Leppaniemi A. Current status and future options for trauma and emergency surgery in Europe. Ulus Travma Acil Cerrahi Derg 2008;14:5-9.

36. Leppäniemi A. A survey on trauma systems and education in Europe. Eur J Trauma Emerg Surg 2008;34:577-81. 